\title{
A New Method for Chebyshev Approximation of Complex-Valued Functions
}

\author{
By K. Glashoff and K. Roleff
}

\begin{abstract}
In this paper we are concerned with a formulation of the Chebyshev approximation problem in the complex plane as a problem of linear optimization in the presence of infinitely many constraints. It is shown that there exist stable and fast algorithms for the solution of optimization problems of this type. Some numerical examples are presented.
\end{abstract}

1. Introduction. We are going to consider the following approximation problem: Let $C(T)$ be the normed space of complex-valued continuous functions $f$ on a compact subset $T$ of the complex plane $\mathbf{C}$, equipped with the uniform norm

$$
\|f\|_{\infty}=\max _{z \in T}|f(z)| \text {. }
$$

Let $f(z)$ and $w_{1}(z), \ldots, w_{n}(z)$ be fixed given functions out of $C(T)$. For any set of $n$ complex parameters $x=\left\{x_{1}, \ldots, x_{n}\right\}$, let

$$
L(x, z)=\sum_{r=1}^{n} x_{r} w_{r}(z)
$$

The problem is to determine an optimal set of parameters $x$ such that

$$
\|f-L(x, z)\|_{\infty} \leqslant\|f-L(x, z)\|_{\infty} \text { for all } x .
$$

Problems of this type appear in many connections and have been treated by various authors; cf. [2], [12], [13]. Considering the computation of best rational Chebyshev approximations to complex-valued functions by descent algorithms, Ellacott and Williams [6] report that the main portion of computer time is spent in the solution of the linear subproblems. They have applied the very slowly convergent Lawson algorithm to these subproblems, and they remark that a fast linear algorithm would bring about a significant improvement in efficiency.

In our paper we describe a new algorithm which seems to be the first one which works for the continuous case (where $T$ is not a finite set), too. In our method we transform the complex approximation problem into a (real) linear optimization problem with infinitely many constraints, a so-called semi-infinite program (SIP); cf. [5], [8], [10]. This is solved by a two-step procedure: in the first step we apply the stabilized Simplex algorithm to a discrete approximation of the SIP and in the second we make use of the Newton-Raphson method in order to obtain very rapidly the solution of the SIP and thus of our original approximation problem.

Received March 23, 1979; revised March 27, 1980.

1980 Mathematics Subject Classification. Primary 65E05; Secondary 30C30. 
2. A Simple Transformation. Let us consider the problem (1) which we restate as an equivalent optimization problem (2) below (here we introduce an additional real variable $\left.y_{0}\right)$ :

(2) Minimize $y_{0}$ under the constraints $|f(z)-L(x, z)|<y_{0}$ for all $z \in T$.

This is a problem of nonlinear optimization, and it has been shown by Krabs and Opfer [12], Barrodale, Delves, and Mason [2] how to solve (2) by means of suitably chosen nonlinear programming codes (if $T$ is a finite set).

Our approach is a different one. We note that the nonlinear constraints of (2) can be restated as a set of linear restrictions. This is true because, for any complex number $z$,

$$
|z|=\max \{\operatorname{Re}(z \eta) / \eta \in S\},
$$

where $S=\{\eta \in \mathrm{C} /|\eta|=1\}$ and where $\operatorname{Re}(w)$ denotes the real part of the complex number $w$. Thus, we can reformulate (2) as the following linear optimization problem:

(3) Minimize $y_{0}, \quad y_{0}+\operatorname{Re}\{L(x, z) \eta\} \geqslant \operatorname{Re}\{f(z) \eta\} \quad$ for all $(\eta, z) \in S \times T$.

This optimization problem has $2 n+1$ real variables, $y_{0}, y_{1}, \ldots, y_{n}, y_{n+1}, \ldots, y_{2 n}$, where we define

$$
x_{k}=y_{k}+i y_{n+k}, \quad k=1, \ldots, n .
$$

Now the restrictions of (3) can be written as

$$
\begin{aligned}
y_{0}+\sum_{r=1}^{n} y_{r} \operatorname{Re}\left\{w_{r}(z) \eta\right\}-\sum_{r=1}^{n} y_{n+r} \operatorname{Im}\left\{w_{r}(z) \eta\right\} \geqslant & \operatorname{Re}\{f(z) \eta\}, \\
& \text { for all }(\eta, z) \in S \times T .
\end{aligned}
$$

Before we consider a simplification of (4), which is possible for most applications, we formulate the dual problem (in the sense of linear optimization, cf. Glashoff and Gustafson [8]):

$$
\begin{aligned}
& \text { Maximize } \sum_{i=1}^{2 n+1} a_{i} \operatorname{Re}\left\{f\left(z_{i}\right) \eta_{i}\right\} \text { subject to the constraints } \\
& \left.\begin{array}{l}
a_{i} \geqslant 0, \quad\left(\eta_{i}, z_{i}\right) \in S \times T(i=1, \ldots, 2 n+1), \\
\sum_{i=1}^{2 n+1} a_{i} \operatorname{Re}\left\{w_{r}\left(z_{i}\right) \eta_{i}\right\}=0 \\
\sum_{i=1}^{2 n+1} a_{i} \operatorname{Im}\left\{w_{r}\left(z_{i}\right) \eta_{i}\right\}=0
\end{array}\right\}, \quad r=1, \ldots, n, \\
& \sum_{i=1}^{2 n+1} a_{i}=1 .
\end{aligned}
$$

Let us now consider the case where $T$ is a simply connected region bounded by a piecewise smooth Jordan curve $C$. Additionally, we assume that the functions $f$ and $w_{r}$ are analytic in the interior of $T$ and continuous on the closure of $T$. Then

$$
\|f\|_{\infty}=\max _{z \in C}|f(z)|
$$

and therefore our problem is equivalent to best approximation of $f(z)$ by $L(x, z)$ on the boundary $C$ of $T$. Let $C$ have the representation 


$$
C=\{z(t) / t \in[0,1]\}, \quad z(0)=z(1) .
$$

Then the optimization problem (3) can be equivalently formulated in the following way (where we use the fact that $S=\{\exp (i s) / s \in[0,2 \pi)\}$ ):

Minimize $y_{0}$ subject to the constraints

$$
\begin{aligned}
y_{0}+ & \sum_{r=1}^{n} y_{r} \operatorname{Re}\left\{w_{r}(z(t)) \exp (i s)\right\}-\sum_{r=1}^{n} y_{n+r} \operatorname{Im}\left\{w_{r}(z(t)) \exp (i s)\right\} \\
& \geqslant \operatorname{Re}\{f(z(t)) \exp (i s)\} \quad \text { for all }(s, t) \in[0,2 \pi) \times[0,1) .
\end{aligned}
$$

Problems of this type (“Semi-infinite Programs", SIP's) appeared first in a paper by Charnes, Cooper, and Kortanek [5]. It is possible to solve (6) by the straightforward generalization of the Simplex method to the case of infinitely many constraints; see for example Carasso [4], Hoffmann and Klostermair [11].

An extension of Remez' second algorithm for certain SIP's is due to Gustafson [9]. Encouraging applications of this algorithm to approximation problems in two dimensions-which are very similar in structure to (6)-are reported by Andreassen and Watson [1]. A computer program (Fahlander [7]) which can be applied directly to (6) is available.

We are not going into the details of the theory and numerics of SIP (cf. [8], [9], [10]) but will just develop the main ideas which are essential for a numerical procedure.

3. Numerical Method and Examples. A very simple, but nevertheless efficient, method for obtaining good approximations of the solution of (6) is the following: instead of the infinite number of inequality constraints appearing in (6), make use of only finitely many of them. This means: choose a fixed finite subset $\Delta_{m}$ of $[0,2 \pi) \times[0,1)$. For example, construct the following 'grid'

$$
\Delta_{m}=\left\{\left(s_{j}, t_{k}\right) / j=1, \ldots, l, k=1, \ldots, p\right\},
$$

where

$$
\begin{aligned}
& s_{j}=2 \pi(j-1) / l, \quad j=1, \ldots, l, \\
& t_{k}=(k-1) / p, \quad k=1, \ldots, p .
\end{aligned}
$$

Here $l$ and $p$ are fixed numbers, and $m=p l$.

Now we consider the following Linear Program derived from (6):

$$
\text { Minimize } y_{0} \text { subject to the constraints }
$$

$$
\sum_{r=0}^{2 n} y_{r} a_{r}\left(s_{j}, t_{k}\right) \geqslant b\left(s_{j}, t_{k}\right) ; \quad j=1, \ldots, l, k=1, \ldots, p .
$$

Here we use the notation

$$
a_{r}(s, t)= \begin{cases}1, & r=0, \\ \operatorname{Re}\left\{w_{r}(z(t)) \exp (i s)\right\}, & r=1, \ldots, n, \\ -\operatorname{Im}\left\{w_{r}(z(t)) \exp (i s)\right\}, & r=n+1, \ldots, 2 n,\end{cases}
$$

and

$$
b(s, t)=\operatorname{Re}\{f(z(t)) \exp (i s)\} .
$$

For our examples, which we describe below, we used a FORTRAN program, evaluating the functions $a_{r}$ and $b$ by means of complex arithmetic. 
We solved the dual of the LP (7) by a stabilized version of the Simplex method due to Bartels and Golub [3] using triangular factorization of basis matrices. It turned out that good approximations were obtained for rather coarse grids very efficiently.

Example 1 (cf. [2]). Here it is required to approximate $f(z)=1 /(z-2)$ on the disc $|z| \leqslant 1$ by a polynomial of degree $n-1$ with real coefficients. In the table below we show three typical results which we obtained using a TR 440 AEG-Telefunken computer (Computer Centre of the University of Hamburg). In all three examples the grid consisted of $l \times p=33 \times 51=1581$ points.

\begin{tabular}{r|ccc}
$n:$ & 3 & 5 & 7 \\
\hline exact $y_{0}:$ & 0.083333 & 0.020833 & 0.005208 \\
\hline$y_{0}$ & 0.083311 & 0.020833 & 0.005207 \\
$y_{1}$ & -0.500018 & -0.499999 & -0.500003 \\
$y_{2}$ & -0.250020 & -0.250001 & -0.249999 \\
$y_{3}$ & -0.166653 & -0.125001 & -0.124996 \\
$y_{4}$ & - & -0.062499 & -0.062505 \\
$y_{5}$ & - & -0.041667 & -0.031249 \\
$y_{6}$ & - & - & -0.015622 \\
$y_{7}$ & - & - & -0.010419 \\
\hline CPU time: & 2.46 & 4.52 & 9.13 \\
$(\mathrm{sec})$ & & &
\end{tabular}

The number of Simplex-iterations (Phase I + Phase II) were $5+8$ for the first example, $9+12$ for the second, and $14+25$ for the third one.

Example 2. Conformal mapping onto the unit circle; cf. [12]. Here it is required to approximate the identity $z$ on the boundary $C$ of a region $T$ by polynomials of higher degree. We treated this problem for a number of regions but just want to present the results for the most difficult of these, namely the $L$-shaped region shown in Figure 1.

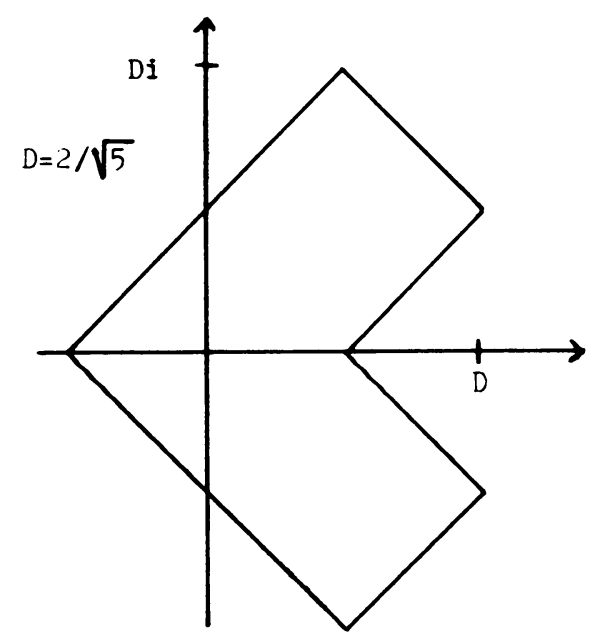

FigURE 1 
Symmetry considerations lead to the conclusion that the imaginary parts of the solution vector must vanish and that it is sufficient to approximate $z$ on the part of the boundary which lies in the upper half plane. Thus, the problem is

$$
\underset{y_{1}, \ldots, y_{n}}{\operatorname{Minimize}} \max _{t \in[0,2]}\left|z(t)-\sum_{r=1}^{n} y_{r} z(t)^{r+1}\right| \text {, }
$$

where

$$
z(t)=2 / \sqrt{5} \cdot \begin{cases}(1 / 2+t)+i t, & 0 \leqslant t \leqslant 1 / 2, \\ (3 / 2-t)+i t, & 1 / 2<t \leqslant 1, \\ (3 / 2-t)+i(2-t), & 1<t<2 .\end{cases}
$$

Choosing $l=17, p=51$, and $n=9$, we obtained the following results:

$$
\begin{aligned}
& y_{1}=0.858, \quad y_{2}=0.159, \quad y_{3}=-3.237, \quad y_{4}=7.201, \\
& y_{5}=-11.01, \quad y_{6}=11.49, \quad y_{7}=-9.039, \quad y_{8}=4.486, \\
& y_{9}=-1.427 .
\end{aligned}
$$

The minimal distance is $y_{0}=0.4332 ; 14+38$ Simplex-iterations were needed ( $7.58 \mathrm{sec}$. CPU-time). The resulting image of the upper part of the $L$-shaped region under the conformal mapping

$$
z \rightarrow z-\sum_{r=1}^{n} y_{r} z^{r+1}
$$

corresponding to our solution, is shown in Figure 2.

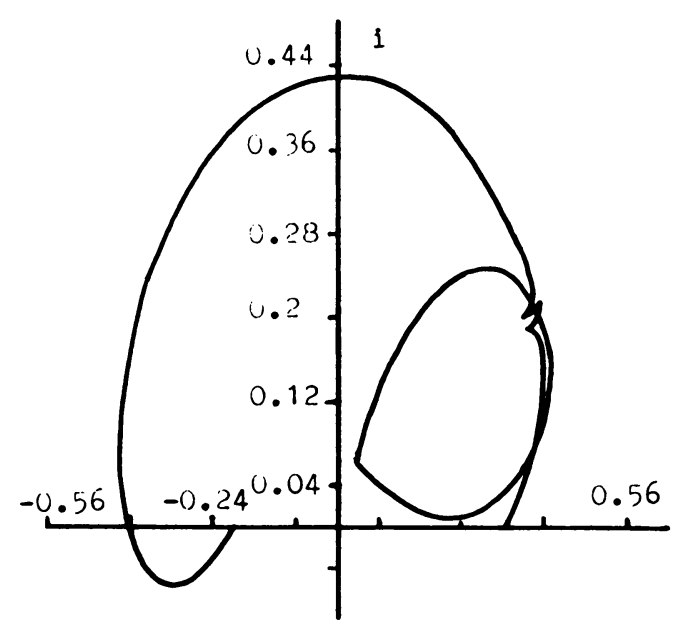

Figure 2

It seems to us that this way of approximately computing conformal mappings for rather complicated domains is not very promising. Increase of $n$ (up to $n=21$ ) gave only very slow improvements of the result.

Example 3. Complex Chebyshev polynomial of degree 8 on a rectangle; cf. [13]. This is the problem of approximating the monomial $z^{8}$ by a polynomial of lower degree. $T$ is chosen as the rectangle $[-2,2] \times[-1,1]$, and because of symmetry 
arguments it is sufficient to consider the following problem:

$$
\text { Minimize } \max _{t \in[0,3]}\left|z(t)^{8}-\sum_{r=1}^{4} y_{r} z(t)^{2(r-1)}\right| \text {, }
$$

where

$$
z(t)= \begin{cases}2+i t, & 0 \leqslant t \leqslant 1 \\ (3-t)+i, & 1<t \leqslant 3\end{cases}
$$

and where the parameters $y_{1}, \ldots, y_{4}$ can be chosen as real numbers in advance. The following table shows some results of our computations for various grids $\Delta_{m}$ with different grid sizes.

The number of Simplex-iterations needed for the solution of the corresponding Linear Programs did not exceed $7+10$ (Phase I + Phase II) and the CPU-time on

\begin{tabular}{|c|c|c|c|c|c|c|c|}
\hline$l$ & $p$ & $m$ & $y_{0}$ & $y_{1}$ & $y_{2}$ & $y_{3}$ & $y_{4}$ \\
\hline 9 & 19 & 171 & 99.93 & -13.91 & 45.78 & -31.55 & 7.69 \\
\hline 9 & 150 & 1350 & 100.13 & -15.08 & 44.76 & -31.52 & 7.76 \\
\hline 17 & 87 & 1479 & 101.91 & -14.79 & 46.60 & -31.80 & 7.72 \\
\hline 34 & 43 & 1462 & 101.88 & -15.18 & 46.09 & -31.86 & 7.75 \\
\hline \multicolumn{3}{|c|}{ 'exact' solution } & 102.12 & -15.17 & 46.33 & -31.88 & 7.75 \\
\hline
\end{tabular}
the TR 440 did not exceed $3.08 \mathrm{sec}$. for any of the four problems.

We have computed other Chebyshev Polynomials (up to degree 10) on various regions of the plane, too. All these examples show the same behavior as the one above, with a total number of Simplex-iterations of maximal $7+15$ on a grid of maximal 1500 points and computing times around $3 \mathrm{sec}$.

We are now going to discuss the last row of the table above where the result of the computation of the 'exact' solution of the continuous problem is shown.

We used the method developed by Gustafson [9], [10]. We cannot describe this method in detail here. Let us just state that its main idea is the following. A nonlinear system of equations is constructed for the 'unknowns' $y_{0}, \ldots, y_{4}$ of the primal problem and the variables of the corresponding dual; cf. (5). In our case, this led to a system of 15 nonlinear equations for 15 variables. We solved this system numerically by Newton's method, using the solution of the discretized problems as starting vectors. Numerical convergence was observed after four iterations.

The absolute value of the optimal error function attains its maximum at the following points $t_{i}$ (with corresponding phases $s_{i}$ ); cf. Figure 3:

$$
\begin{array}{ll}
t_{1}=0.3163 & s_{1}=5.1345 \\
t_{2}=1 & s_{2}=1.2494 \\
t_{3}=1.7954 & s_{3}=3.4424 \\
t_{4}=3 & s_{4}=0
\end{array}
$$




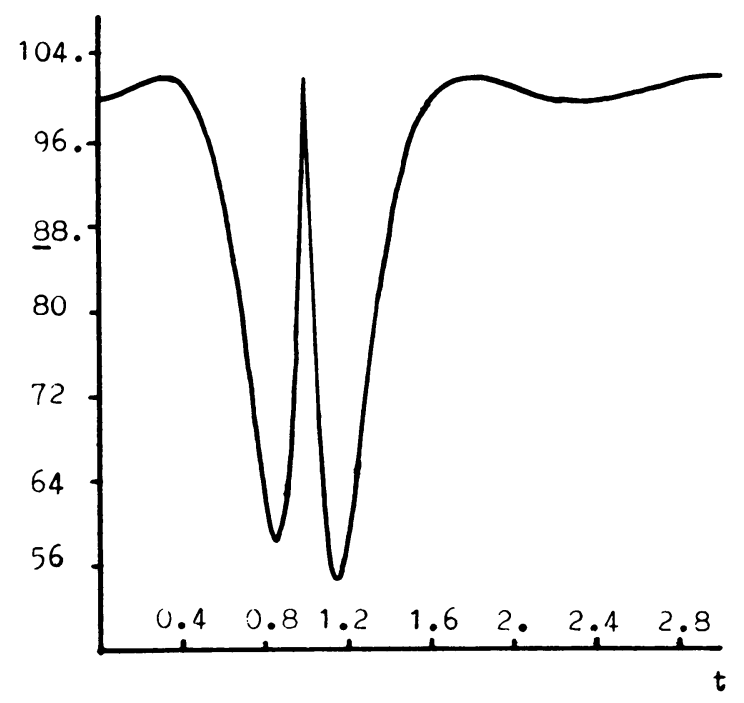

Figure 3

Institut für Angewandte Mathematik

Universität Hamburg

D-2000 Hamburg 13, West Germany

Siemens AG

D-8000 München 70, West Germany

1. D. O. Andreassen \& G. A. Watson, "Linear Chebyshev approximation without Chebyshev sets," $B I T$, v. 16, 1976, pp. 349-362.

2. I. Barrodale, L. M. Delves \& J. C. Mason, "Linear Chebyshev approximation of complexvalued functions," Math. Comp., v. 32, 1978, pp. 853-863.

3. R. H. BARTels \& G. H. GOLUB, "The simplex method of linear programming using $L U$-decompositions," Comm. ACM, v. 12, 1969, pp. 266-268.

4. C. Carasso, L'Algorithme d'Echange en Optimisation Convexe, These, Grenoble, 1973.

5. A. Charnes, W. W. Cooper \& K. O. Kortanek, "Duality, Haar programs and finite sequence spaces," Proc. Nat. Acad. Sci. U. S. A., v. 48, 1962, pp. 783-786.

6. S. Ellacott \& J. Williams, "Rational Chebyshev approximation in the complex plane," SIAM J. Numer. Anal., v. 13, 1976, pp. 310-323.

7. K. FAhlander, Computer Programs for Semi-Infinite Optimization, TRITA-NA-7312, Department of Numerical Analysis, Royal Institute of Technology, S-10044, Stockholm 70, Sweden, 1973.

8. K. Glashoff \& S. A. Gustafson, Einführung in die lineare Optimierung, Wissenschaftliche Buchgesellschaft, Darmstadt, 1978.

9. S. A. Gustafson, "On the computational solution of a class of generalized moment problems," SIAM J. Numer. Anal., v. 7, 1970, pp. 343-357.

10. S. A. Gustafson, "Nonlinear system in semi-infinite programming," in Numerical Solution of Nonlinear Algebraic Systems (G. B. Byrnes \& C. A. Hall, Eds.), Academic Press, New York, 1973, pp. 63-99.

11. K.-H. Hoffmann \& A. KlostermaiR, "A semi-infinite linear programming procedure and applications to approximation problems in optimal control," Approximation Theory II, Proc. Internat. Sympos., Austin, Texas, 1976, pp. 379-389.

12. W. Krabs \& G. OpFer, "Eine Methode zur Lösung des komplexen Approximationsproblems mit einer Anwendung auf konforme Abbildungen," Z. Angew. Math. Mech., v. 55, 1975, pp. 208-211.

13. G. OPFER, "An algorithm for the construction of best approximations based on Kolmogorov's criterion,” J. Approx. Theory, v. 23, 1978, pp. 299-317. 